\title{
Using Polymer Hydrogels for Glyphosate Sequestration from Aqueous Solutions: Molecular Theory Study of Adsorption to Polyallylamine Films
}

\author{
Néstor A. Pérez-Chávez, Alberto G. Albesa, and Gabriel S. Longo*(i) \\ Instituto de Investigaciones Fisicoquímicas Teóricas y Aplicadas (INIFTA), UNLP-CONICET, Diag. 113 y 64 S/N, B1904 La Plata, \\ Argentina
}

\section{Supporting Information}

\begin{abstract}
A molecular theory has been applied to study the equilibrium conditions of glyphosate and aminomethylphosphonic acid (AMPA) adsorption from aqueous solutions to hydrogel films of cross-linked polyallylamine (PAH). This theoretical framework allows for describing the size, shape, state of charge/protonation, and configurational freedom of all chemical species in the system. Adsorption of glyphosate is a nonmonotonic function of the solution $\mathrm{pH}$, which results from the protonation behavior of both the adsorbate and adsorbent material. Glyphosate and chloride ions compete for adsorption to neutralize the polymer charge; lowering the solution salt concentration enhances the partition of

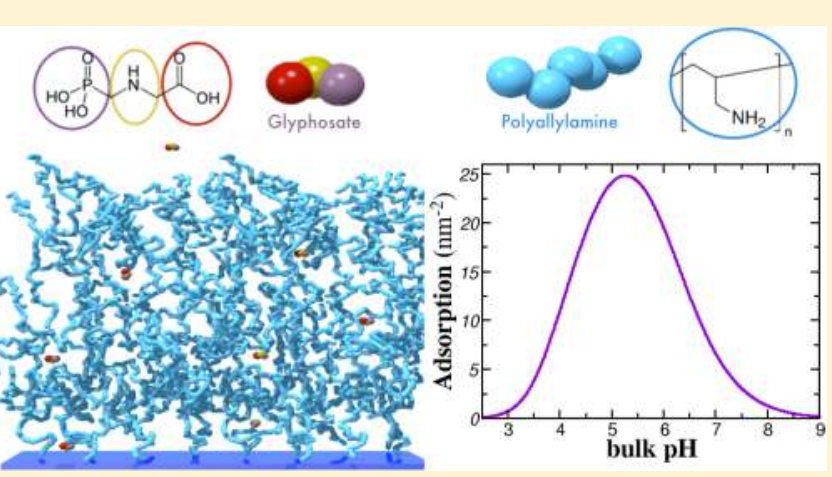
glyphosate inside the hydrogel film. AMPA adsorption is qualitatively similar to that of glyphosate but orders of magnitude smaller under the same conditions. AMPA is less charged than glyphosate, which unbalances the competition for adsorption with salt counter ions. In mixed solutions, glyphosate presence can significantly hinder AMPA adsorption. A higher $\mathrm{pH}$ establishes inside the film than in the bulk solution, which has important implications for the herbicide biodegradation because microbial activity is $\mathrm{pH}$-dependent. Thus, $\mathrm{PAH}$ hydrogel films can be considered as functional materials that combine glyphosate sequestration and in situ degradation. In devising these materials, the polymer density is an important variable of design; polymer networks with high density of titratable units can enhance adsorption; this density can also be used to modify the $\mathrm{pH}$ inside the material.
\end{abstract}

\section{INTRODUCTION}

Glyphosate (GLP) is the world's best-selling and most widely applied pesticide. ${ }^{1,2}$ In Argentina, GLP use continues to increase with 0.2 megatons consumed in 2012, which represents $80 \%$ of total herbicide sales in the country. ${ }^{3}$ This efficient broadspectrum herbicide, used nonselectively in agriculture, works by blocking a biochemical pathway leading to the production of essential amino acids, causing plant death by starvation. ${ }^{4}$ Because humans do not produce essential amino acids, GLP use is presumed safe. However, its efficiency as a weed killer, its nontoxicity, and the engineering of genetically modified crops that can resist GLP exposure have, in many cases, led to excessive use in the control of weeds and grasses. GLP has been detected in different soils, groundwaters, surface waters, and its bottom sediments, not only in those used for agriculture; ${ }^{5-7}$ it has also been found in processed food, drinking water, and pharmaceutical products. ${ }^{8,9}$ Therefore, it is nowadays clear that development of means for GLP removal is of critical importance.

Current methods of GLP removal from aqueous solutions include chemical precipitation, ${ }^{10}$ advanced oxidation, ${ }^{11,12}$ membrane filtration, ${ }^{13}$ biodegradation, ${ }^{14}$ and physical adsorption. ${ }^{15-18}$ Methods involving physical adsorption are generally efficient, low cost, and more environmentally friendly, producing no secondary pollution. ${ }^{15}$ Different adsorbent materials have been considered for GLP sequestration, including resins, ${ }^{15}$ graphene oxides, ${ }^{16}$ starch, ${ }^{17}$ and zeolites. ${ }^{18}$ In all those studies, GLP adsorption is due to electrostatic attractions with the substrate. These interactions are highly modulated by environmental conditions, such as the $\mathrm{pH}$ and salt concentration. Indeed, several experimental studies show the importance of $\mathrm{pH}$ and salt concentration in the physical adsorption of GLP to different materials. ${ }^{15,17,18}$

Therefore, understanding the physical and chemical properties that govern pesticide adsorption and their coupling with environment composition is essential in the development of materials with optimal contaminant removal/sequestration capacity. In this work, we investigate the adsorption of GLP, AMPA, and their mixtures to grafted poly(allylamine hydrochloride) (PAH) cross-linked networks. We are particularly interested in the role that environment $\mathrm{pH}$ and salt concentration have in determining the adsorption behavior. To this goal, we have applied a molecular-level theory that allows

Received: August 10, 2018

Revised: September 16, 2018

Published: September 24, 2018 
for describing size, shape, state of protonation, and conformations of all of the chemical species in the system.

Degradation of GLP in soils and waters occurs mainly because of bacterial activity. ${ }^{19,20}$ In one of the paths for biodegradation, an oxidase breaks down the carbon-nitrogen bond of GLP, which produces aminomethylphosphonic acid (AMPA), the most abundant byproduct of this herbicide. ${ }^{20}$ However, when the medium $\mathrm{pH}$ is not optimal, bacterial activity slows down, which reduces degradation efficiency and results in longer environmental persistence. ${ }^{20}$ Our goal is to show that PAH films can combine enhanced GLP sequestration with a higher $\mathrm{pH}$ environment in the interior of the hydrogel, which provides optimal conditions for biodegradation.

\section{METHOD}

To study the adsorption of GLP (and AMPA) to hydrogel films of grafted cross-linked PAH chains, we apply a molecular-level theory with explicit description of all chemical species that compose the system. In this approach, the protonation state of each of the different titratable units of GLP is not assumed but predicted as a result of the local interplay between the freeenergy cost of protonation/deprotonation, the entropic loss of molecular confinement, the conformational degrees of freedom of the network and the adsorbate, and the electrostatic and steric interactions. This is achieved through the formulation of a general free energy that includes all of these contributions. This molecular theory was originally developed to study adsorption of histidine peptides to grafted polyacid networks ${ }^{21}$ and has been recently extended to investigate protein protonation upon adsorption to such $\mathrm{pH}$-responsive hydrogel films. ${ }^{22,23}$ The method is based on an extension of the theory developed by Nap et al. ${ }^{24}$ and Gong et al. ${ }^{25}$ to consider the behavior of grafted weak polyelectrolyte layers. Next, we outline the theoretical method used in this study, whereas a full description can be found in our recent work. $^{23}$

The system consists of an aqueous solution in contact with a network of cross-linked polyallylamine that is chemically grafted to a planar surface (see Figure 1). The coordinate $z$ measures the distance from the surface placed at $z=0$. The solution is composed of water molecules, hydronium and hydroxide ions,

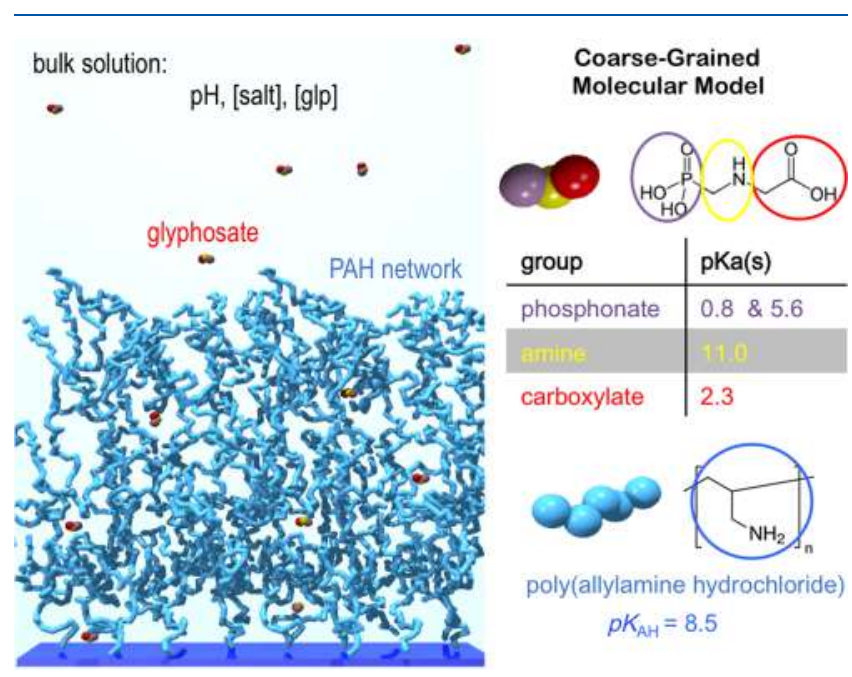

Figure 1. Left: Scheme representing the adsorption of GLP from a salt solution to a surface-grafted, cross-linked PAH network. The coarsegrained molecular model is illustrated on the right. AMPA is modeled similarly to GLP but without including the carboxylate unit. and a monovalent salt $(\mathrm{NaCl})$ that is assumed to be completely dissociated into chloride and sodium ions. This solution can additionally contain a finite concentration of either GLP, AMPA, or both molecules; we will refer to these species as the adsorbates.

The Helmholtz free energy of the system can be expressed as

$$
\begin{aligned}
F= & -T S_{\mathrm{cnf}, \mathrm{nw}}+F_{\mathrm{chm}, \mathrm{nw}}-T S_{\mathrm{mix}} \\
& +\sum_{i}\left(-T S_{\mathrm{cnf}, i}-T S_{\mathrm{tr}, i}+F_{\mathrm{chm}, i}\right) \\
& +U_{\mathrm{st}}+U_{\mathrm{vdW}}+U_{\text {elect }}
\end{aligned}
$$

where the first term in the right-hand side of this expression includes the conformational entropy of the network $\left(S_{\mathrm{cnf}, \mathrm{nw}}\right)$, which is due to the many possible spatial distributions of $\mathrm{PAH}$ segments (conformations), and $T$ represents the system temperature. The second term is the chemical free energy of the network $\left(F_{\text {chm,nw }}\right)$ that accounts for the acid-base equilibrium of PAH segments. The next term includes the translational and mixing entropy of all free species, except the adsorbates, as well as the formation self-energies of these species. In the second line of eq 1, the sum runs over GLP and/or AMPA if they are present in the solution; for a particular adsorbate $(i \in$ $\{$ GLP, AMPA $\}$ ), these terms include its conformational entropy $\left(S_{\mathrm{cnf}, i}\right)$, its translational entropy and self-energy $\left(S_{\mathrm{tr}, i}\right)$, and the chemical free energy $\left(F_{\mathrm{chm}, i}\right)$ that results from the acid-base equilibrium of its titratable units. The third line in eq 1 includes the energetic contributions to the free energy, incorporating steric (excluded volume) repulsions $\left(U_{\text {st }}\right)$, van der Waals attractions $\left(U_{\mathrm{vdW}}\right)$, and electrostatic interactions $\left(U_{\text {elect }}\right)$. This system is in equilibrium with a bulk solution, which fixes the chemical potentials of all free species. As a result, the proper thermodynamic potential whose minimum yields equilibrium is the semigrand potential, which is a function of these chemical potentials. The semigrand potential results from the Legendre transform of the Helmholtz free energy (eq 1).

The aforementioned contributions to the free energy can be explicitly written in terms of a few functions: (i) the local densities of all free species; (ii) the local densities of different configurations of the adsorbates; (iii) the probability distribution of network conformations; (iv) the local degrees of protonation of all titratable species including network and adsorbate units; and (v) the local electrostatic potential. The next step in this method is the optimization, with respect to each of these functions, of the semigrand potential. Such a procedure allows for expressing functions (i) to (iv) in terms of only two position-dependent interaction potentials: the local osmotic pressure and the electrostatic potential.

These local interaction potentials can be numerically calculated through iteratively solving both the Poisson equation and the incompressibility constraint imposed to the fluid. This last restriction assures that at each position, the available volume is fully occupied by some of the chemical species. After these interaction potentials are determined, any thermodynamic quantity of interest can be derived from the free energy. At this point, the local functions that compose the free energy are known as well, which allows for the calculation of different local and average quantities.

\section{MOLECULAR MODEL}

A molecular model of all chemical species must be defined to apply this theory and calculate results. GLP is represented using 
a coarse-grained model, where we include the phosphonate, amine, and carboxylate groups. The molecular volumes assigned to these coarse-grained units, as well as to other species in the system, are presented in Table 1 . To construct this coarse-

Table 1. Molecular Volumes of the Coarse-Grained Units Considered in Our Model

\begin{tabular}{lc}
\multicolumn{1}{c}{ species/group } & volume \\
$\mathrm{H}_{2} \mathrm{O}, \mathrm{OH}^{-}, \mathrm{H}_{3} \mathrm{O}^{+}$ & $0.030 \mathrm{~nm}^{3}$ \\
salt ions $\left(\mathrm{Na}^{+}, \mathrm{Cl}^{-}\right)$ & $0.033 \mathrm{~nm}^{3}$ \\
phosphonate & $0.054 \mathrm{~nm}^{3}$ \\
amine & $0.040 \mathrm{~nm}^{3}$ \\
carboxylate & $0.056 \mathrm{~nm}^{3}$ \\
allylamine & $0.068 \mathrm{~nm}^{3}$
\end{tabular}

grained model, we have performed density functional theory $(\mathrm{DFT})^{26}$ geometry optimizations using Gaussian 03 package; ${ }^{27}$ the B3LYP hybrid exchange-correlation functional ${ }^{28,29}$ and the $6-31 G(d, p)$ basis set were used. All geometrical parameters were optimized without constraints. The coarse-grained units are assigned to the position of the carbon atom in the carboxylate group, the nitrogen in the amine, and the phosphorus in the phosphonate group. These positions are kept fixed with respect to the center of mass of the GLP molecule according to the DFT optimization of the atomistic structure. The molecule, however, has full rotational and translational freedom. An AMPA molecule is represented in the same way but without including the carboxylate unit.

The $\mathrm{p} K_{\mathrm{a}}$ values of titratable units have been obtained from the literature. ${ }^{30-33}$ For GLP, these values are 0.8 and 5.6 for the diacidic phosphonate group, 2.3 for the carboxylic acid, and 11.0 for the basic amine group; ${ }^{30,32}$ for AMPA, we have used 1.8 and 5.4 for the phosphonate and 10.0 for the amine group. ${ }^{30,32}$ In the case of the basic allylamine segments of the network, their $\mathrm{p} K_{\mathrm{a}}$ is $8.5 .^{31,33}$ For the self-dissociation of water, we use $\mathrm{p} K_{\mathrm{w}}=14$.

Using this $\mathrm{p} K_{\mathrm{a}}$ scheme, Figure 2 shows the charge of GLP and AMPA in dilute solution as a function of $\mathrm{pH}$. The plot also

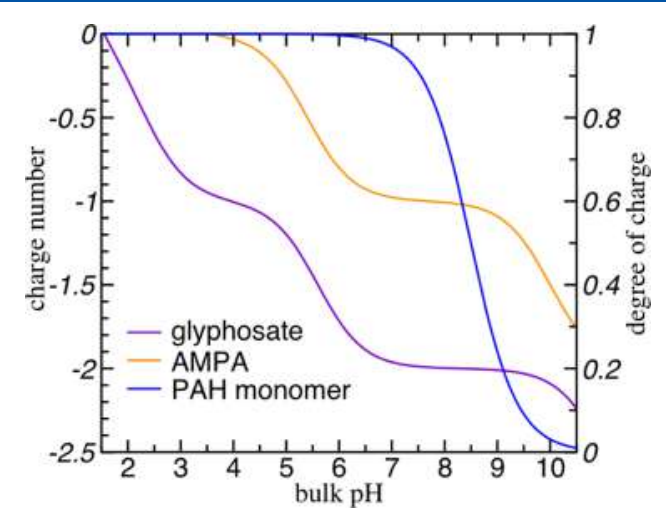

Figure 2. Plot of average GLP and AMPA charge in dilute solution as a function of $\mathrm{pH}$ (left-hand side $y$-axis). The right-hand side $y$-axis corresponds to the degree of protonation of the isolated allylamine monomer under the same conditions.

includes the degree of charge of an isolated allylamine monomer under the same conditions. Given that adsorption to the PAH film is expected to be driven by adsorbate-network electrostatic attractions, these curves anticipate a nonmonotonic $\mathrm{pH}$ dependence of such adsorption. At low $\mathrm{pH}$, the PAH segments will be strongly positively charged, but GLP or AMPA adsorption will bring less net charge to the film than the adsorption of a (smaller) chloride ion. Similarly at high $\mathrm{pH}$, GLP and AMPA are more negatively charged, but $\mathrm{PAH}$ segments will only be weakly charged. Only for intermediate $\mathrm{pH}$ values, where both adsorbent and adsorbate are strongly charged, we expect significant adsorption to occur.

The different molecular conformations of the PAH network are also an input needed to evaluate this theory. This network is composed of cross-linked 50-segment long polymer chains, where each segment is a coarse-grained representation of a PAH unit, having $0.5 \mathrm{~nm}$ segment length. Most of these chains connect two cross-linking segments, except those top-most chains, which have their solution-side ends free, and some chains that are connected by one of their ends to a surface-grafted segment. The cross-linking units have coordination four, and the structure has a diamond-like topology. ${ }^{34-37}$ To generate network conformations, we have performed molecular dynamics (MD) simulations using GROMACS 5.1.2, ${ }^{38-40}$ where the network is a periodic molecule composed of 30 cross-linking segments, 2 grafting points, and 64 chains with a total of 3200 PAH units. The supporting surface in the MD simulation box has a $33 \mathrm{~nm}^{2}$ area; periodic boundary conditions are imposed in both the $x$ and $y$ directions. The force field used in this MD simulation has been well described in other works. ${ }^{34,36,41,42}$

To numerically solve the equations resulting from the molecular theory, the Poisson equation, and the incompressibility constraint, space is discretized into $0.5 \mathrm{~nm}$ thick layers parallel to the supporting surface (the $x-y$ plane). The system is assumed to be isotropic in the $x$ and $y$ directions. The aqueous medium has a dielectric constant $\epsilon_{\mathrm{w}} \epsilon_{0}$, where $\epsilon_{\mathrm{w}}=78.5$ is the relative dielectric constant of water at room temperature and $\epsilon_{0}$ is the permittivity of vacuum.

\section{RESULTS AND DISCUSSION}

We will first consider the effect of solution $\mathrm{pH}$ and salt concentration on the adsorption of GLP to PAH films. To quantify such a behavior, we define the adsorption as the number of molecules adsorbed per unit area, in excess of the bulk contribution. Namely

$$
\Gamma=\int_{0}^{\infty} \mathrm{d} z\left(\rho_{\mathrm{glp}}(z)-\rho_{\mathrm{glp}}^{\text {bulk }}\right)
$$

where $\rho_{\mathrm{glp}}(z)$ and $\rho_{\mathrm{glp}}^{\mathrm{bulk}}$ are the local and bulk (number) densities of GLP, respectively, such that $\lim _{z \rightarrow \infty} \rho_{\text {glp }}(z)=\rho_{\text {glp }}^{\text {bulk }}$. Note that $\Gamma$ includes contributions from adsorbed molecules throughout the whole thickness of the hydrogel film as well as from the polymer-solution interface.

Figure 3 describes the adsorption of GLP as a function of the bulk solution composition. Panel A shows that $\Gamma$ is a decreasing function of the salt concentration, which is a clear signature that adsorption is driven by PAH-GLP electrostatic attractions. An increasing salt concentration makes the adsorption of chloride ions more favorable, which results in the screening of these attractions, diminishing the capacity of the film to adsorb the herbicide. Panel B of Figure 3 shows that GLP adsorption is a nonmonotonic function of the solution $\mathrm{pH}$, a result that can be explained by looking at the charge behavior of both species, the adsorbate and adsorbent material, displayed in Figure 2. At low $\mathrm{pH}$, most segments of the polymer network are charged, but GLP is mostly in its electroneutral state; chloride ions will preferentially adsorb as counter ions under such conditions. At high $\mathrm{pH}$, on the other hand, the situation reverses: while GLP is negatively charged, polymer network units are deprotonated 

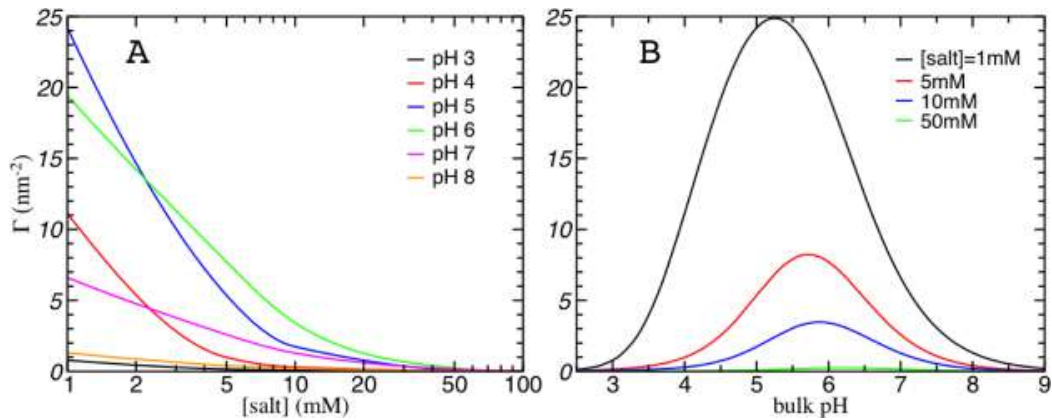

Figure 3. Plots of GLP adsorption, $\Gamma$, as a function of the bulk solution composition: panel A shows $\Gamma$ as a function of the salt concentration for different $\mathrm{pH}$ values; panel $\mathrm{B}$ shows the adsorption as a function of the $\mathrm{pH}$ for various salt concentrations. In both panels, the concentration of the adsorbate in the bulk solution is $[\mathrm{glp}]=10 \mu \mathrm{M}$.

(uncharged). As a result, negligible adsorption occurs at both extremes of the $\mathrm{pH}$ scale. In the intermediate $\mathrm{pH}$ range, where both adsorbent and adsorbate are strongly and oppositely charged, significant adsorption takes place, leading to the nonmonotonic curves observed in Figure 3B.

The general trends of the behavior predicted in Figure 3 when changing the solution composition have been experimentally found for GLP adsorption to other materials. Guo et al. ${ }^{17}$ have recently highlighted the critical importance of $\mathrm{pH}$ and $\mathrm{NaCl}$ concentration in the adsorption of GLP from aqueous solutions to cross-linked amino-starch. Their work shows decreasing adsorption with increasing salinity as well as a nonmonotonic $\mathrm{pH}$-dependent adsorption with a maximum around $\mathrm{pH}$ 6. In addition, Zhou et al. ${ }^{15}$ have shown that the adsorption of GLP from water to nanosized microporous exchange resins, modified with copper hydroxide, depends nonmonotonically on the $\mathrm{pH}$ having a peak around $\mathrm{pH} 6-7$. This $\mathrm{pH}$ dependence has also been reported upon GLP adsorption to zeolites. ${ }^{18}$ In these different systems, the nonmonotonic behavior can be understood with a slight modification of our previous argument using the $\mathrm{pH}$ dependence of the bulk solution charge. However, we will show that such reasoning to understand GLP adsorption can be misleading in other situations, in particular because GLP deprotonation upon adsorption plays a critical role in defining adsorption to PAH films.

In both panels of Figure 3, maximum adsorption of GLP is achieved near $\mathrm{pH}$ 5. The net charge (number) of the molecule is approximately -1 at this $\mathrm{pH}$ (see Figure 2 ), the same as chloride ions. This raises the question of what drives the adsorption behavior observed in Figure 3 because the adsorption of the more abundant $([$ salt $]>[\mathrm{glp}])$, smaller chloride ion is expected. To address this question, Figure 4 shows different local quantities as a function of the distance to the supporting surface at $\mathrm{pH} 5$ for both low and high salt concentrations. Panel A shows the PAH volume fraction under such conditions; this curve is not very sensitive to an increase in the salt concentration, so we only include the case $[$ salt $]=1 \mathrm{mM}$. We find that the polymer network extends up to $h \approx 130 \mathrm{~nm}$ from the surface; below this distance $(z<h)$ that defines the thickness of the hydrogel film, PAH segments occupy roughly $5 \%$ of the local volume.

Panel B of Figure 4 shows the local concentration of GLP. This graph shows that the behavior described in Figure 3 is not a surface phenomenon, but the adsorbate partitions at different spatial regions throughout the whole thickness of the hydrogel film. Inside the film, this concentration increases several orders of magnitude with respect to the bulk value $(10 \mu \mathrm{M})$. Consistent with the results of Figure 3, lowering the salt concentration decreases the local concentration of GLP inside the film. At 1 $\mathrm{mM}$ salt, GLP is mainly responsible for neutralizing the polymer charge, whereas increasing the availability of the salt from the solution to $10 \mathrm{mM}$ results in chloride ions being the most abundant adsorbed counter ions.

When in contact with these relatively low ionic strength solutions, the film only adsorbs enough counter ions to neutralize the electric charge of network segments. ${ }^{22}$ This is because of the high cost that results from the loss of translational entropy when these counter ions are confined inside the film. In this context, the adsorption behavior of Figure $4 \mathrm{~B}$ can be interpreted by considering panel $\mathrm{C}$ that shows the average, local net charge of GLP. A few nanometers above the top film surface, the average charge of the molecule becomes that of the bulk solution; most GLP molecules are in the -1 charge state because its average charge is close to that value. Inside the film, however, the GLP charge is around -2. Under the conditions of Figure 4, adsorbing one GLP molecule brings more counter ion charge to the film for the entropic price of confining just one molecule. This explains why the film adsorbs GLP which, in the solution, is much less abundant than chloride ions and similarly charged. As mentioned, increasing salt concentration lowers the entropic cost of confining such ions, which displaces the balance to favor chloride adsorption, as seen in Figure 4B.

The reason for GLP charge regulation can be explained considering panel D of Figure 4, which shows the local $\mathrm{pH}$. Sufficiently far from the film $z \gg h \approx 130 \mathrm{~nm}$, local $\mathrm{pH}$ takes the bulk value. Inside the film, however, a much higher $\mathrm{pH}$ establishes; the local $\mathrm{pH}$ increases 2-3 units in the particular cases of Figure 4. Upon adsorption, the higher-pH environment results in the deprotonation of GLP acidic units, which explains the more negative charge of the molecule inside the film. This increase in $\mathrm{pH}$ not only favors adsorption but has also the additional advantage of potentially enhancing biodegradation of the pesticide inside the film. It has been reported that nonneutral $\mathrm{pH}$ slows down microbial activity, lowering the efficiency in GLP biodegradation. ${ }^{20}$

In addition, local $\mathrm{pH}$ is relevant because this single quantity provides information on the local state of charge of all ionizable species, including the different units of GLP and those of the polymer network. Panel A of Figure 5 shows the film $\mathrm{pH}, \mathrm{pH}_{\mathrm{pol}}$, calculated as an average from $z=0$ up to $z=h$, as a function of the bulk pH. Film $\mathrm{pH}$, which depends on the solution salt concentration, is higher than the $\mathrm{pH}$ imposed to the bulk solution, in almost all the scale. One of the consequences of this last result, combined with those shown in Figure 3B, is that PAH films offer a wide range of conditions that can simultaneously 

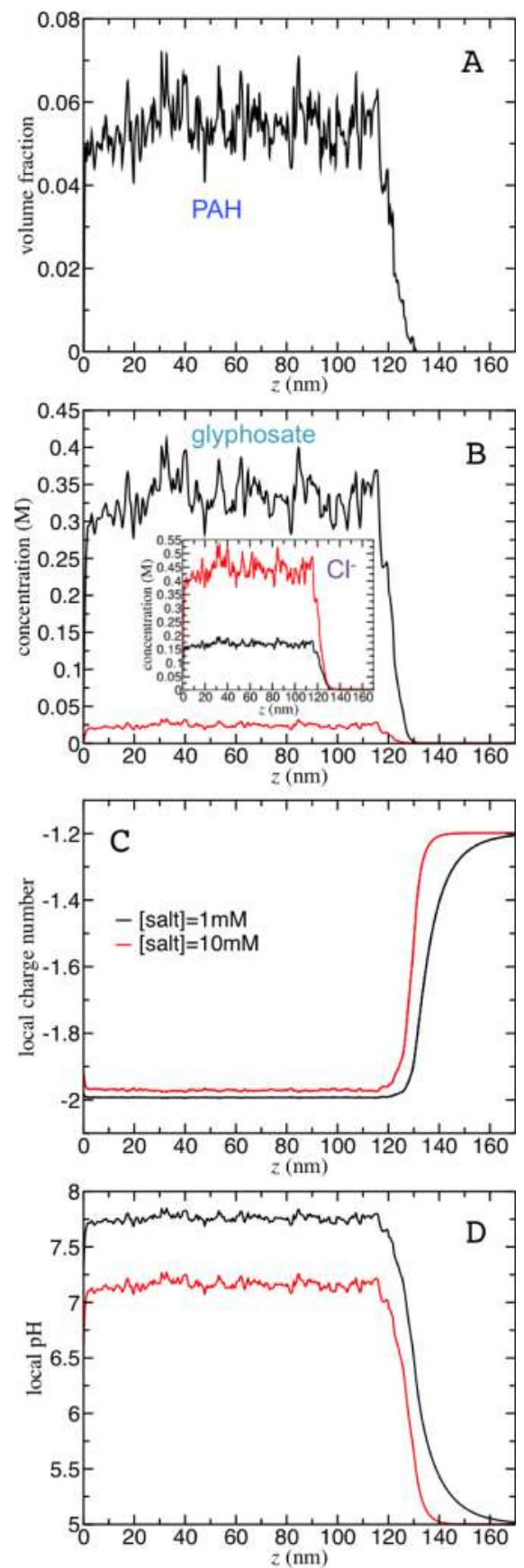

Figure 4. Plots of different local quantities at $\mathrm{pH} 5$ and two salt concentrations, $1 \mathrm{mM}$ (black curves) and $10 \mathrm{mM}$ (red curves): local polymer volume fraction (A), local GLP concentration (B), net charge (C), and local $\mathrm{pH}$ (D) all as a function of the distance to the supporting surface, $z$. The inset in panel B shows the local concentration of chloride ions at the same conditions. The bulk concentration of GLP is [glp] = $10 \mu \mathrm{M}$.

drive adsorption and result in more favorable conditions for degradation inside the film.

Because $\mathrm{pH}$ is higher inside the film, the average charge of adsorbed GLP is more negative, which is shown in Figure 5B. This behavior depends on the salinity of the solution; the lower the salt concentration, the more negatively charged the adsorbate is. The maximum deviation from the bulk solution conditions occurs near $\mathrm{pH} 5$; the conditions are also shown in Figure 4. As we have briefly mentioned, the more negative
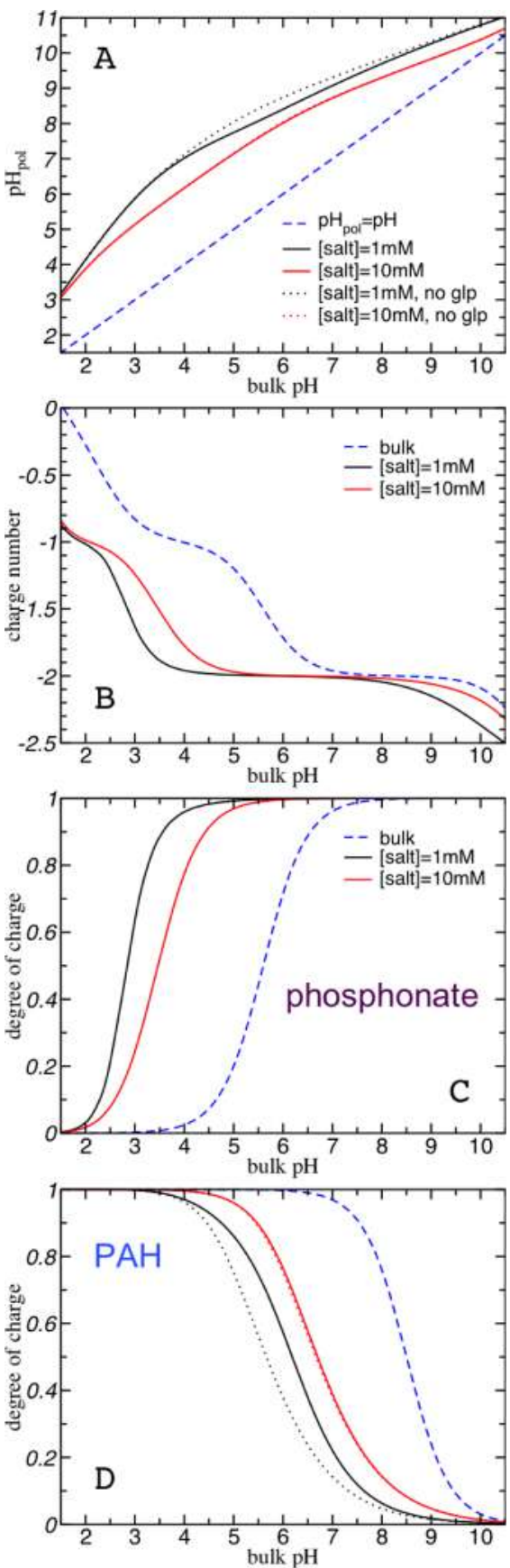

Figure 5. Plots showing the $\mathrm{pH}$-dependence of different quantities at two salt concentrations, $1 \mathrm{mM}$ (black-line curves) and $10 \mathrm{mM}$ (red-line curves), and $[\mathrm{glp}]=10 \mu \mathrm{M}$. (A) Average $\mathrm{pH}$ inside the film, $\mathrm{pH}_{\mathrm{pol}}$ (dashed-line curve corresponds to $\mathrm{pH}_{\mathrm{pol}}=\mathrm{pH}$ ); the dotted lines show $\mathrm{pH}_{\text {pol }}$ for solutions containing no GLP. (B) Average net charge of adsorbed GLP molecules (dashed-line curve shows the solution charge); (C) average degree of charge of phosphonate groups corresponding to adsorbed GLP molecules (dashed-line curve corresponds to solution molecules); (D) average degree of protonation of network segments for solutions with (solid line) and without (dotted line) GLP (dashed-line curve represents the protonation of the isolated monomer in dilute solution).

charge of GLP inside the film is due to deprotonation of its acidic units inside the film resulting from the higher $\mathrm{pH}$ environment. To illustrate this behavior, Figure 5C shows the degree of charge of phosphonate units corresponding to adsorbed GLP molecules as a function of bulk $\mathrm{pH}$. Compared to the bulk 
solution, inside the film, this unit is significantly more

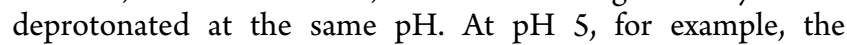
phosphonate unit is mostly protonated (uncharged) in the solution, whereas inside the film, it is fully deprotonated (charged) at both salt conditions shown in the plot. The magnitude of this displacement toward deprotonation, however, does depend on the salt concentration because the film $\mathrm{pH}$ also shows this dependence (see Figure 5A).

The higher $\mathrm{pH}$ inside the film also results in the deprotonation of PAH units; this behavior is presented in Figure 5D. Namely, compared to the isolated monomer, these units are significantly more deprotonated (less charged) when confined to the polymer network, which depends on the salt concentration. In the absence of GLP, this behavior occurs to reduce electrostatic repulsions between like-charged polymer units. When GLP is in the bulk solution, this allows the polymer to be more protonated, although protonation is still much lower than the ideal behavior (see Figure 5D), particularly at low salt concentration.

In this context, knowing the film $\mathrm{pH}$ provides information about the state of charge of all species inside the film. For example, using the intrinsic $\mathrm{p} K_{\mathrm{a}}$ of its units, one can construct Figure 2 and evaluate it at the film $\mathrm{pH}$ (rather than the bulk $\mathrm{pH}$ ), which gives a reasonable approximation of the molecule charge inside the film. However, film $\mathrm{pH}$ results from the nontrivial balance between all of the physicochemical contributions to the free energy. Let us consider the following situation: a higher film $\mathrm{pH}$ allows for the adsorbate to be more strongly charged inside the hydrogel than in the solution, favoring adsorption due to stronger electrostatic attractions. This higher film $\mathrm{pH}$, however, results in the deprotonation of network units lowering their degree of charge, which weakens the electrostatic attractions and disfavors adsorption. For both species, the chemical free-energy cost of deprotonation favors a lower $\mathrm{pH}$, equal to the bulk value. Therefore, the $\mathrm{pH}$ that establishes inside the film results from the conditions that yield thermodynamic equilibrium; such conditions emerge from the complex local interplay between all physical interactions, chemical equilibrium, and molecular organization at the environmental conditions imposed externally (bulk solution composition). Experimental access to this quantity requires very dilute concentrations of the probe; we have shown in Figure 5A that adsorption, even at low bulk concentrations $([\mathrm{glp}]=10 \mu \mathrm{M})$, can modify the $\mathrm{pH}$ inside the material.

We now investigate the effect of polymer density on the adsorption of GLP to PAH hydrogel films. To address this question, we have considered three films with the same topology but having different polymer densities. The volume fraction of these networks is shown at in Figure $6 \mathrm{~A}$ as a function of the distance to the supporting surface. Figure $6 \mathrm{~B}$ shows the $\mathrm{pH}$ dependence of GLP adsorption to these hydrogel films. Although the films have similar thickness, increasing the polymer density enhances adsorption per unit area. This enhancement is due to a higher negative charge density that occurs in the films as the density of PAH increases. The degree of protonation, however, decreases as the volume fraction increases, an effect that is compensated by the higher density of protonable PAH segments (see Supporting Information).

Moreover, given the solution $\mathrm{pH}$, Figure $6 \mathrm{~B}$ inset shows that the film $\mathrm{pH}$ changes with the network density. As the polymer density increases, so does the $\mathrm{pH}$ inside the material. In particular, for the cases presented in Figure 6, $\mathrm{pH}_{\mathrm{pol}} 7$ occurs within the range of $\mathrm{pH}$ values $3.5-5$. This behavior suggests that polymer density can be used as a design parameter not only to
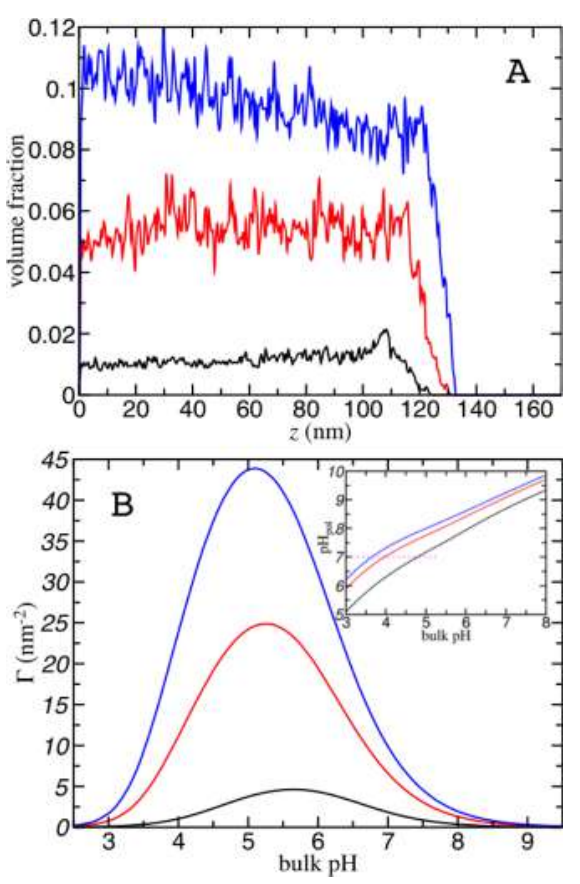

Figure 6. (A) Polymer volume fraction as a function of the distance to the supporting surface for three different networks at $\mathrm{pH}$ 5. (B) GLP adsorption as a function of $\mathrm{pH}$ for the three different polymer films of panel $\mathrm{A}$; the inset shows the $\mathrm{pH}$ inside the film. In both panels, the conditions are $[$ salt $]=1 \mathrm{mM}$ and $[\mathrm{glp}]=10 \mu \mathrm{M}$.

enhance adsorption but also to achieve optimal degradation conditions inside the material.

Next, we consider how GLP adsorption depends on its bulk concentration. Adsorption isotherms are presented in Figure 7 for different $\mathrm{pH}$ values and salt concentrations. Here, we see again that the effect of increasing salt concentration is reducing the effective capacity of the hydrogel to adsorb GLP. Moreover, the $\mathrm{pH}$ of maximum adsorption depends sensibly on the GLP concentration as well as the salt concentration. In the Supporting Information, we compare the results of Figure 7, obtained with our molecular theory, with well-known, simple adsorption isotherm models.

AMPA is the most abundant degradation product of GLP. Thus, in Figure 8, we consider the adsorption of AMPA to the $\mathrm{PAH}$ film in terms of the composition of the bulk solution. These curves show the same general trends of the adsorption of GLP seen in Figure 3. AMPA adsorption decreases with increasing salt concentration because of the enhancing role of chloride ions to neutralize the network charge (see Figure $8 \mathrm{~B}$ ). Moreover, adsorption of AMPA is a nonmonotonic function of the bulk $\mathrm{pH}$ (see Figure 8B). At low pH, AMPA is only weakly charged (see Figure 2), whereas the polymer is weakly charged at high $\mathrm{pH}$. Under these conditions, no significant adsorption occurs. At intermediate $\mathrm{pH}$, however, both molecules are strongly charged, leading to the maximum in the $\mathrm{pH}$-dependent adsorption profiles of Figure 8B.

The adsorption of GLP and that of AMPA are qualitatively similar. However, the adsorption of GLP is roughly 2 orders of magnitude larger than that of AMPA at the same conditions (compare Figures 3 and 8). The reason for this behavior is the additional carboxylate group that GLP bears. Under most conditions, this group is deprotonated when GLP adsorbs, which means that this molecule bears one more negative charge inside the hydrogel (see Supporting Information). 

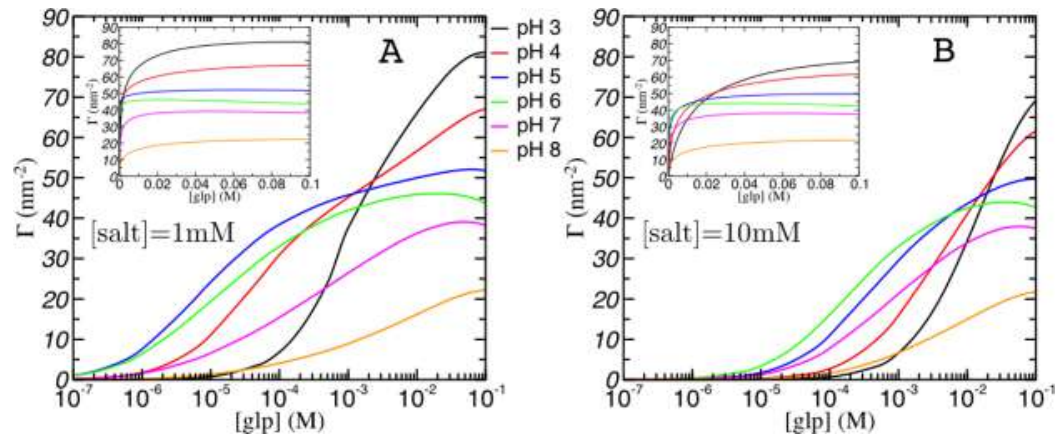

Figure 7. Adsorption isotherms: plot of $\Gamma$ as a function of the solution concentration of GLP. The various curves correspond to different $\mathrm{pH}$ values. The salt concentrations of panel A and B are 1 and $10 \mathrm{mM}$, respectively. The insets show the same curves using a linear $\mathrm{x}$-scale.
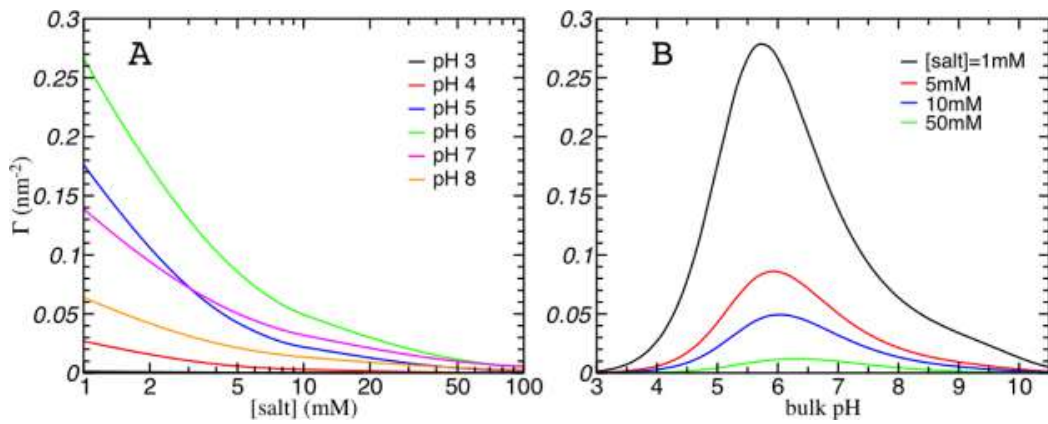

Figure 8. Plot of AMPA adsorption, $\Gamma$, as a function of the bulk solution composition: panel A shows $\Gamma$ as a function of the salt concentration for different $\mathrm{pH}$ values; panel $\mathrm{B}$ shows the adsorption as a function of the $\mathrm{pH}$ for various salt concentrations. The concentration of the adsorbate in the bulk solution is $[\mathrm{ampa}]=10 \mu \mathrm{M}$.

In this context, the next question that we address is how is the adsorption from mixtures of GLP and AMPA? Figure 9 shows the adsorption from such mixtures as a function of $\mathrm{pH}$ at
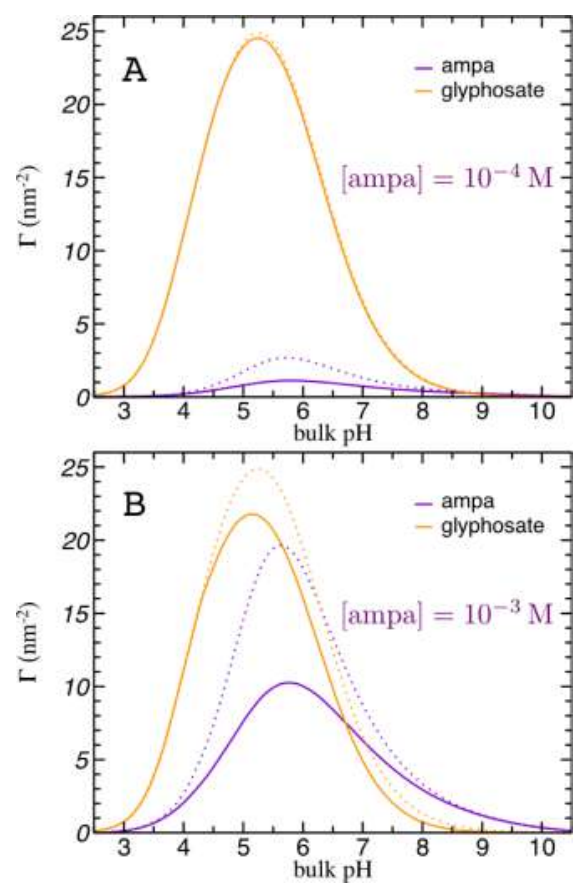

Figure 9. Adsorption from mixtures of GLP and AMPA as a function of the $\mathrm{pH}$, for solutions having [ampa] $=10^{-4} \mathrm{M}$ (panel $\left.\mathrm{A}\right)$ or $[\mathrm{ampa}]=$ $10^{-3} \mathrm{M}$ (panel B). In both panels, [glp] $=10^{-5} \mathrm{M}$ and [salt $]=1 \mathrm{mM}$. The dotted-line curves show the adsorption from pure solutions at otherwise the same conditions. relatively low salt conditions. In panel A, the bulk concentration of AMPA is 1 order of magnitude larger than that of GLP. Under these conditions, the adsorption of GLP from pure solutions is more than an order of magnitude larger than the adsorption of AMPA from pure solutions. As a consequence, the adsorption of GLP from mixtures is not affected by the presence of AMPA. Contrarily, GLP reduces the adsorption of AMPA to approximately half of the value for pure solutions (in the $\mathrm{pH}$ range around maximum adsorption). When increasing the bulk concentration of AMPA, the situation is qualitatively similar, as seen in Figure 9B. Although the adsorption of GLP in the mixture diminishes with respect to pure solutions, the main effect is on the adsorption of AMPA. Under these conditions that are a priori more favorable for AMPA adsorption, GLP significantly reduces the adsorption of AMPA when comparing mixtures to pure solutions.

\section{CONCLUSIONS}

In this work, we have applied a molecular-level theory to study the equilibrium adsorption to grafted $\mathrm{PAH}$ hydrogel films from salt solutions containing GLP, AMPA, or both. Our molecular model includes description of size, shape, conformation, and charge state of all chemical species, including the adsorbates and the polymer network. We have focused our attention in the role that solution composition ( $\mathrm{pH}$, salt, and adsorbate concentration) has on the adsorption behavior. Adsorption of GLP (or AMPA) is a nonmonotonic function of the solution $\mathrm{pH}$, which is explained in terms of the charging behavior of both the polymer network and adsorbate. The $\mathrm{pH}$ of maximum adsorption depends on the salt and adsorbate concentrations. Our predictions show that in finding the optimal conditions for adsorption, salt concentration is a critical variable. The lower the 
salinity of the medium, the more GLP (or AMPA) adsorbs to the film. In particular, a sufficiently low salt concentration allows for a wide range of $\mathrm{pH}$ values where adsorption is significant.

Therefore, the optimal conditions for adsorption are achieved lowering the salt concentration. The reason for this behavior is the competitive adsorption between GLP and salt anions. These ions adsorb to the film to neutralize the charge in the polymer. Counter ion confinement has an entropic cost associated with the loss of translational entropy. As a counter ion, GLP is only slightly better than a monovalent anion; depending on the $\mathrm{pH}$, this herbicide bears an additional negative charge, which favors its adsorption. Such adsorption brings the same counter ion charge to film but at a lower entropic cost of molecular confinement. However, GLP adsorption has also some disadvantages with respect to salt anion adsorption; first, it is a larger molecule and second, the additional charge requires some of its titratable groups to be deprotonated, which increases the chemical free energy that describes this acid-base equilibrium. As a result, the ratio between GLP and salt concentrations becomes a critical factor in deciding the winner in this competitive adsorption game.

Inside the film, a higher $\mathrm{pH}$ establishes than the bulk value. The main effect of this phenomenon is that the adsorbate regulates its charge. Upon adsorption, GLP is more negatively charged than in the bulk solution, which enhances electrostatic attractions with the positively charged network and drives adsorption. Equally important, this increase in $\mathrm{pH}$ has implications for GLP biodegradation because microbial activity depends on the acidity of the medium. Therefore, PAH films offer an additional advantage to pesticide sequestration from aqueous solutions. Namely, under some experimental conditions, biodegradation inside the material can be enhanced.

AMPA is the GLP main metabolite. In pure solutions, the adsorption of GLP is significantly higher than that of AMPA at the same conditions. This occurs because GLP bears an additional carboxylate group that deprotonates as the herbicide adsorbs. For this reason, GLP can hinder the adsorption of AMPA from binary mixtures. This behavior points to an additional advantage of PAH films for GLP sequestration and biodegradation. Although biodegradation occurs inside this material, GLP will remain adsorbed.

Finally, in devising PAH films for GLP sequestration and degradation, the material density of the polymer is an important design variable. We have shown that polymer networks with high density of titratable units can enhance adsorption. In addition, the film $\mathrm{pH}$ can be modified using the network density if the goal is to optimize in situ microbial degradation.

\section{ASSOCIATED CONTENT}

\section{S Supporting Information}

The Supporting Information is available free of charge on the ACS Publications website at DOI: 10.1021/acs.langmuir.8b02727.

Additional results and adsorption isotherms (PDF)

\section{AUTHOR INFORMATION}

\section{Corresponding Author}

*E-mail: longogs@inifta.unlp.edu.ar.

\section{ORCID $\odot$}

Gabriel S. Longo: 0000-0001-8353-5163

Notes

The authors declare no competing financial interest.

\section{ACKNOWLEDGMENTS}

This work was supported by CONICET and ANPCyT (PICT2014-3377), Argentina. N.A.P.-C. acknowledges a ANPCyT fellowship (PICT-2015-3425).

\section{REFERENCES}

(1) Woodburn, A. T. Glyphosate: Production, pricing and use worldwide. Pest Manag. Sci. 2000, 56, 309-312.

(2) Benbrook, C. M. Trends in glyphosate herbicide use in the United States and globally. Environ. Sci. Eur. 2016, 28, 1-15.

(3) Primost, J. E.; Marino, D. J. G.; Aparicio, V. C.; Costa, J. L.; Carriquiriborde, P. Glyphosate and AMPA, "pseudo-persistent" pollutants under real-world agricultural management practices in the Mesopotamic Pampas agroecosystem, Argentina. Environ. Pollut. 2017, 229, 771-779.

(4) Martinez, D. A.; Loening, U. E.; Graham, M. C. Impacts of glyphosate-based herbicides on disease resistance and health of crops: a review. Environ. Sci. Eur. 2018, 30, 2.

(5) Ronco, A. E.; Marino, D. J. G.; Abelando, M.; Almada, P.; Apartin, C. D. Water quality of the main tributaries of the Paraná Basin: Glyphosate and AMPA in surface water and bottom sediments. Environ. Monit. Assess. 2016, 188, 458.

(6) Rendon-von Osten, J.; Dzul-Caamal, R. Glyphosate Residues in Groundwater, Drinking Water and Urine of Subsistence Farmers from Intensive Agriculture Localities: A Survey in Hopelchén, Campeche, Mexico. Int. J. Environ. Res. Public Health 2017, 14, 595.

(7) Castro Berman, M.; Marino, D. J. G.; Quiroga, M. V.; Zagarese, H. Occurrence and levels of glyphosate and AMPA in shallow lakes from the Pampean and Patagonian regions of Argentina. Chemosphere 2018, 200, 513-522.

(8) Younes, M.; Galal-Gorchev, H. Pesticides in drinking water-A case study. Food Chem. Toxicol. 2000, 38, S87-S90.

(9) World Health Organization Glyphosate and AMPA in DrinkingWater, 2005.

(10) Liu, Z.; Zhu, M.; Yu, P.; Xu, Y.; Zhao, X. Pretreatment of membrane separation of glyphosate mother liquor using a precipitation method. Desalination 2013, 313, 140-144.

(11) Lan, H.; He, W.; Wang, A.; Liu, R.; Liu, H.; Qu, J.; Huang, C. P. An activated carbon fiber cathode for the degradation of glyphosate in aqueous solutions by the Electro-Fenton mode: Optimal operational conditions and the deposition of iron on cathode on electrode reusability. Water Res. 2016, 105, 575-582.

(12) Xing, B.; Chen, H.; Zhang, X. Efficient degradation of organic phosphorus in glyphosate wastewater by catalytic wet oxidation using modified activated carbon as a catalyst. Environ. Technol. 2018, 39, $749-758$.

(13) Song, J.; Li, X.-M.; Figoli, A.; Huang, H.; Pan, C.; He, T.; Jiang, B. Composite hollow fiber nanofiltration membranes for recovery of glyphosate from saline wastewater. Water Res. 2013, 47, 2065-2074.

(14) Loperena, L.; Ferrari, M. D.; Saravia, V.; Murro, D.; Lima, C.; Ferrando, L.; Fernández, A.; Lareo, C. Performance of a commercial inoculum for the aerobic biodegradation of a high fat content dairy wastewater. Bioresour. Technol. 2007, 98, 1045-1051.

(15) Zhou, C.; Jia, D.; Liu, M.; Liu, X.; Li, C. Removal of glyphosate from aqueous solution using nanosized copper hydroxide modified resin: Equilibrium isotherms and kinetics. J. Chem. Eng. Data 2017, 62, 3585-3592.

(16) Santos, T. R. T.; Andrade, M. B.; Silva, M. F.; Bergamasco, R.; Hamoudi, S. Development of $\alpha$ - and $\gamma$-Fe2O3 decorated graphene oxides for glyphosate removal from water. Environ. Technol. 2017, 3330, $1-20$.

(17) Guo, L.; Cao, Y.; Jin, K.; Han, L.; Li, G.; Liu, J.; Ma, S. Adsorption characteristics of glyphosate on cross-linked amino-starch. J. Chem. Eng. Data 2018, 63, 422-428.

(18) Zavareh, S.; Farrokhzad, Z.; Darvishi, F. Modification of zeolite $4 \mathrm{~A}$ for use as an adsorbent for glyphosate and as an antibacterial agent for water. Ecotoxicol. Environ. Saf. 2018, 155, 1-8. 
(19) Hallas, L. E.; Adams, W. J.; Heitkamp, M. A. Glyphosate degradation by immobilized bacteria: Field studies with industrial wastewater effluent. Appl. Environ. Microbiol. 1992, 58, 1215-1219.

(20) la Cecilia, D.; Maggi, F. Analysis of glyphosate degradation in a soil microcosm. Environ. Pollut. 2018, 233, 201-207.

(21) Longo, G. S.; Olvera de la Cruz, M.; Szleifer, I. Equilibrium adsorption of hexahistidine on $\mathrm{pH}$-responsive hydrogel nanofilms. Langmuir 2014, 30, 15335-15344.

(22) Longo, G. S.; Szleifer, I. Adsorption and protonation of peptides and proteins in $\mathrm{pH}$ responsive gels. J. Phys. D. Appl. Phys. 2016, 49, 323001.

(23) Hagemann, A.; Giussi, J. M.; Longo, G. S. The Use of pH Gradients in Responsive Polymer Hydrogels for the Separation and Localization of Proteins from Binary Mixtures. Macromolecules [Online early access.] DOI: 10.1021/acs.macromol.8b01876. Published Online: October 9, 2018. http://dx.doi.org/10.1021/acs.macromol. 8 b01876.

(24) Nap, R.; Gong, P.; Szleifer, I. Weak polyelectrolytes tethered to surfaces: Effect of geometry, acid-base equilibrium and electrical permittivity. J. Polym. Sci. Part B Polym. Phys. 2006, 44, 2638-2662.

(25) Gong, P.; Genzer, J.; Szleifer, I. Phase behavior and charge regulation of weak polyelectrolyte grafted layers. Phys. Rev. Lett. 2007, 98, 018302.

(26) Kohn, W.; Sham, L. J. Self-consistent equations including exchange and correlation effects. Phys. Rev. 1965, 140, A1133-A1138.

(27) Frisch, M. J.; Trucks, G. W.; Schlegel, H. B.; Scuseria, G. E.; Robb, M. A.; Cheeseman, J. R.; Montgomery, J. A., Jr.; Vreven, T.; Kudin, K. N.; Burant, J. C.; Millam, J. M.; Iyengar, S. S.; Tomasi, J.; Barone, V.; Mennucci, B.; Cossi, M.; Scalmani, G.; Rega, N.; Petersson, G. A.; Nakatsuji, H.; Hada, M.; Ehara, M.; Toyota, K.; Fukuda, R.; Hasegawa, J.; Ishida, M.; Nakajima, T.; Honda, Y.; Kitao, O.; Nakai, H.; Klene, M.; Li, X.; Knox, J. E.; Hratchian, H. P.; Cross, J. B.; Adamo, C.; Jaramillo, J.; Gomperts, R.; Stratmann, R. E.; Yazyev, O.; Austin, A. J.; Cammi, R.; Pomelli, C.; Ochterski, J. W.; Ayala, P. Y.; Morokuma, K.; Voth, G. A.; Salvador, P.; Dannenberg, J. J.; Zakrzewski, V. G.; Dapprich, S.; Daniels, A. D.; Strain, M. C.; Farkas, O.; Malick, D. K.; Rabuck, A. D.; Raghavachari, K.; Foresman, J. B.; Ortiz, J. V.; Cui, Q.; Baboul, A. G.; Clifford, S.; Cioslowski, J.; Stefanov, B. B.; Liu, G.; Liashenko, A.; Piskorz, P.; Komaromi, I.; Martin, R. L.; Fox, D. J.; Keith, T.; Al-Laham, M. A.; Peng, C. Y.; Nanayakkara, A.; Challacombe, M.; Gill, P. M. W.; Johnson, B.; Chen, W.; Wong, M. W.; Gonzalez, C.; Pople, J. A. Gaussian 03, Revision B.03; Gaussian, Inc.: Pittsburgh, PA, 2003.

(28) Lee, C.; Yang, W.; Parr, R. G. Development of the Colle-Salvetti correlation-energy formula into a functional of the electron density. Phys. Rev. B: Condens. Matter Mater. Phys. 1988, 37, 785-789.

(29) Becke, A. D. Density-functional thermochemistry. III. The role of exact exchange. J. Chem. Phys. 1993, 98, 5648-5652.

(30) Sadi, B. B. M.; Vonderheide, A. P.; Caruso, J. A. Analysis of phosphorus herbicides by ion-pairing reversed-phase liquid chromatography coupled to inductively coupled plasma mass spectrometry with octapole reaction cell. J. Chromatogr. A 2004, 1050, 95-101.

(31) Bhatia, S. R.; Khattak, S. F.; Roberts, S. C. Polyelectrolytes for cell encapsulation. Curr. Opin. Colloid Interface Sci. 2005, 10, 45-51.

(32) Chen, Z.; He, W.; Beer, M.; Megharaj, M.; Naidu, R. Speciation of glyphosate, phosphate and aminomethylphosphonic acid in soil extracts by ion chromatography with inductively coupled plasma mass spectrometry with an octopole reaction system. Talanta 2009, 78, 852856.

(33) Lawrence, P. G.; Lapitsky, Y. Ionically cross-linked poly(allylamine) as a stimulus-responsive underwater adhesive: Ionic strength and $\mathrm{pH}$ effects. Langmuir 2015, 31, 1564-1574.

(34) Mann, B. A.; Holm, C.; Kremer, K. Swelling of polyelectrolyte networks. J. Chem. Phys. 2005, 122, 154903.

(35) Quesada-Pérez, M.; Maroto-Centeno, J. A.; Martín-Molina, A. Effect of the counterion valence on the behavior of thermo-sensitive gels and microgels: A Monte Carlo simulation study. Macromolecules 2012, 45, 8872-8879.
(36) Košovan, P.; Richter, T.; Holm, C. Modeling of polyelectrolyte gels in equilibrium with salt solutions. Macromolecules 2015, 48, 76987708 .

(37) Hofzumahaus, C.; Hebbeker, P.; Schneider, S. Monte Carlo simulations of weak polyelectrolyte microgels: $\mathrm{pH}$-dependence of conformation and ionization. Soft Matter 2018, 14, 4087-4100.

(38) Berendsen, H. J. C.; van der Spoel, D.; van Drunen, R. GROMACS: A message-passing parallel molecular dynamics implementation. Comput. Phys. Commun. 1995, 91, 43-56.

(39) Van Der Spoel, D.; Lindahl, E.; Hess, B.; Groenhof, G.; Mark, A. E.; Berendsen, H. J. C. GROMACS: Fast, flexible, and free. J. Comput. Chem. 2005, 26, 1701-1718.

(40) Abraham, M. J.; Murtola, T.; Schulz, R.; Páll, S.; Smith, J. C.; Hess, B.; Lindahl, E. GROMACS: High performance molecular simulations through multi-level parallelism from laptops to supercomputers. SoftwareX 2015, 1-2, 19-25.

(41) Kremer, K.; Grest, G. S. Dynamics of entangled linear polymer melts: A molecular-dynamics simulation. J. Chem. Phys. 1990, 92, $5057-5086$

(42) Longo, G. S.; de la Cruz, M. O.; Szleifer, I. Molecular theory of weak polyelectrolyte gels: The role of $\mathrm{pH}$ and salt concentration. Macromolecules 2011, 44, 147-158. 